\title{
Les dictionnaires bilingues des maîtres ou professeurs auteurs de manuels pédagogiques, J. Lillo (éd.)
}

\section{Monica Barsi}

\section{(2) OpenEdition}

1 Journals

\section{Edizione digitale}

URL: http://journals.openedition.org/studifrancesi/10630

DOI: 10.4000/studifrancesi. 10630

ISSN: 2421-5856

\section{Editore}

Rosenberg \& Sellier

\section{Edizione cartacea}

Data di pubblicazione: 1 décembre 2017

Paginazione: 599-600

ISSN: 0039-2944

\section{Notizia bibliografica digitale}

Monica Barsi, «Les dictionnaires bilingues des maitres ou professeurs auteurs de manuels pédagogiques, J. Lillo (éd.)», Studi Francesi [Online], 183 (LXI | III) | 2017, online dal 01 février 2018, consultato il 21 janvier 2021. URL: http://journals.openedition.org/studifrancesi/10630 ; DOI: https://doi.org/10.4000/ studifrancesi. 10630

Questo documento è stato generato automaticamente il 21 janvier 2021.

\section{(c)}

Studi Francesi è distribuita con Licenza Creative Commons Attribuzione - Non commerciale - Non opere derivate 4.0 Internazionale. 


\title{
Les dictionnaires bilingues des maîtres ou professeurs auteurs de manuels pédagogiques, J. Lillo (éd.)
}

\author{
Monica Barsi
}

\section{NOTIZIA}

Les dictionnaires bilingues des maîtres ou professeurs auteurs de manuels pédagogiques, éd. Jacqueline LILLO, «Documents pour l'histoire du français langue étrangère ou seconde» 56, juin 2016, 216 pp.

1 In questo numero dei «Documents pour l'histoire du français langue étrangère ou seconde» che arricchisce la storia della lessicografia bilingue, l'iniziativa promossa da Jacqueline LILLo è quella di studiare gli autori di dizionari che hanno anche composto manuali per l'insegnamento delle lingue e in particolare della lingua francese, come per esempio le grammatiche contrastive e, spesso con testo a fronte, antologie e prontuari per la corrispondenza. In quasi tutte le pubblicazioni citate la traduzione è la chiave comune per impadronirsi in prima battuta di morfosintassi e lessico, in seconda di diverse terminologie, ampie varietà di registro e molteplici situazioni comunicative. Nell'introduzione (pp. 5-26) la curatrice offre una panoramica sulla storia dell'insegnamento soprattutto ottocentesco, visto attraverso i suoi registi - maestri e professori - e i cosiddetti 'materiali', manuali e dizionari di varie tipologie destinati al pubblico scolastico. I contributi che seguono ruotano attorno a personalità e volumi che fanno parte di una fittissima rete di scambi per lo più tra la Francia e l'Italia ma anche tra la Francia e altri paesi europei (qui solo segnalati).

2 Antonella AMATUZzi si sofferma sulle opere di un notissimo insegnante di italiano, francese e tedesco, oltre che autore di successo di manuali di lingua e dizionari del XVII secolo: Nathanaël Duez auteur du "Guidon de la langue italienne" (1641) et du "Dittionario Francese Italiano" (1659-1660): un maître de langues entre continuité et innovation (pp. 27-50). 
Del Guidon de la langue italienne, grammatica della lingua italiana redatta in francese e pubblicata dal 1641 al 1685, ritraccia la composizione e le fonti; del Dittionario Francese Italiano, utilizzato nei Paesi Bassi e pubblicato dal 1659-60 al 1678, analizza la derivazione dalle Recherches italiennes di Antoine Oudin e le specificità soprattutto pedagogiche. Francesco Paolo Alexandre MADONIA in Un intellectuel exilé dans la tourmente du Risorgimento: Antonio Ronna, lexicographe et professeur (pp. 51-73) illustra il percorso di Antonio Ronna (1801-1866), lessicografo e professore espatriato in Francia per sfuggire al regime austriaco in epoca risorgimentale. Continuatore del dizionario di Biagioli e di quello di Barberi, è autore, nel 1858, di un suo Dictionnaire italien-français, français-italien improntato a quello di Biagioli. Questi diversi repertori, che trasferiscono in Francia molta parte della cultura italiana, sono pubblicati da attivissimi editori quali Charles Hingray e Louis-Claude Baudry. Sempre per Hingray, Ronna pubblica un manuale per l'insegnamento dell'italiano messo a confronto con il francese, il Guide de la conversation français-italien à l'usage des Voyageurs et des Etudiants, che viene tradotto in altre sei lingue e di cui vengono preparate anche un'edizione quadrilingue, un'altra trilingue e delle versioni bilingui. I libri di lettura che divulgano autori come Tasso e Manzoni presso gli scolari francesi completano la produzione di questo prolifico autore. Annick FARINA e Marie-France MERGER si concentrano su uno dei più rinomati lessicografi vissuto tra i due secoli scorsi, Candido Ghiotti (1841-1915). Il titolo del loro contributo riporta quello dell'opera maggiore del Ghiotti: Lexicographie bilingue et didactique: le "Nuovo vocabolario comparativo delle lingue italiana e francese" de Candido Ghiotti (pp. 75-101). Non solo quest'opera, pubblicata nel 1890 da Bellardi e Appiotti, è analizzata anche sulla scorta di studi precedenti, compiuti in particolare da Merger, ma è ricordato anche il diffusissimo Vocabolario scolastico, edito da Petrini nel 1889, e la Grammatica ragionata della lingua francese del 1868, testo scolastico altrettanto utilizzato. Discorso linguistico e discorso più ampiamente culturale, come quello sulla civilisation francese portata a modello nell'Italia post-unitaria, sono al centro dello studio delle due autrici. Michela MURANO descrive la brillante carriera di Giovanni Battista Melzi in Francia nelle pagine intitolate «In comodissimo volume, un gran tesoro di nozioni»: le "Nuovo dizionario francese-italiano e italiano-francese" (1886) du professeur Melzi (pp. 125-148). Insegnante e direttore dell'Ecole des langues modernes a Parigi, Melzi traduce e scrive libri di testo per i Frères Garnier. Si contano, oltre al dizionario bilingue citato nel titolo del contributo, venti manuali di corrispondenza bilingue in tedesco, inglese, spagnolo, francese e italiano distribuiti in Europa e in America del Sud grazie ad accordi editoriali che rivelano una rete fittissima (Ollendorff in Francia, Dulau in Inghilterra, Bocca in Italia, Bouret in America del Sud, Verdager in Spagna, Pierer in Germania). La collaborazione al Grand Dictionnaire Universel du XIX siècle di Pierre Larousse, come esperto per le voci sull'Italia, è altrettanto degna di nota anche perché la colossale opera francese servirà in parte al Melzi per il suo più modesto Nuovo Vocabolario Universale della lingua italiana, pubblicato nel 1880 da Garnier. In Italia, sarà poi autore di dizionari bilingui per la collana dei Dizionari scolastici di Treves. Lo studio di Mariangela ALBANO sulla fraseologia nelle opere di Caricati, La phraséologie dans la grammaire française et le dictionnaire bilingue d'Augusto Caricati (pp. 169-187), aggiunge un nuovo tassello alla storia di questo materiale lessicografico, assolutamente capitale nella glottodidattica. Gli altri contributi che non includono l'italiano ma il francese come lingua straniera sono quelli di Franz-Joseph MEISSNER, La découverte du français non-conventionnel outreRhin: le dictionnaire des "Parisismen" de Césaire Villatte (1816-1895) (pp. 103-124), di Nathalie 
VAN DER SANDEN-PIVA, Les dictionnaires des enseignants auteurs de manuels de français aux Pays-Bas au XIX siècle, nouveaux outils scolaires au service de l'apprentissage des langues (pp. 149-167) e di Manuel BRUÑa CUEVAS, Rafael Reyes et son dictionnaire français-espagnol et espagnol-français (1926-1928) (pp. 189-206).

4 Tutto il corpus linguistico preso in esame appartiene a una forma di trasmissione del sapere attraverso una sistematizzazione che ci porta a riscoprire le conoscenze condivise da intere generazioni in un nutrito confronto tra due culture. Notissime al momento della pubblicazione, le opere destinate alle scuole appartengono a un patrimonio che viene ciclicamente rinnovato e progressivamente dimenticato. Nel susseguirsi di riedizioni, revisioni, nuove edizioni e vere e proprie novità editoriali si può tuttavia cogliere il riflesso di un'avventura culturale di cui gli articoli di questa raccolta ci restituiscono tutta la vitalità. 